Network Working Group

Request for Comments: 3811

Category: Standards Track
T. Nadeau, Ed.

Cisco Systems, Inc.

J. Cucchiara, Ed.

Marconi Communications, Inc.

June 2004

\title{
Definitions of Textual Conventions (TCs) for Multiprotocol Label Switching (MPLS) Management
}

Status of this Memo

This document specifies an Internet standards track protocol for the Internet community, and requests discussion and suggestions for improvements. Please refer to the current edition of the "Internet Official Protocol Standards" (STD 1) for the standardization state and status of this protocol. Distribution of this memo is unlimited.

Copyright Notice

Copyright (C) The Internet Society (2004).

Abstract

This memo defines a Management Information Base (MIB) module which contains Textual Conventions to represent commonly used Multiprotocol Label Switching (MPLS) management information. The intent is that these TEXTUAL CONVENTIONS (TCs) will be imported and used in MPLS related MIB modules that would otherwise define their own representations.

Table of Contents

1. Introduction. . . . . . . . . . . . . . . . . . . . . . . . 2

2. The Internet-Standard Management Framework. . . . . . . . . 2

3. MPLS Textual Conventions MIB Definitions. • • • • • • • • • 2

4. References. . . . . . . . . . . . . . . . . . . . . 16 4.1. Normative References. . . . . . . . . . . . . . 16 4.2. Informative References. . . . . . . . . . . . . . 17

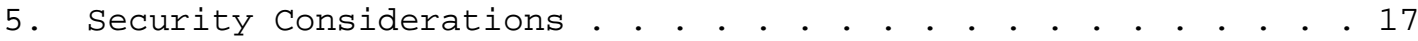

6. IANA Considerations . . . . . . . . . . . . . . . . . . . . . .

7. Contributors. . . . . . . . . . . . . . . . . . . . . 18

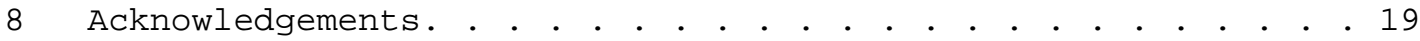

9. Authors' Addresses. . . . . . . . . . . . . . . . . . . . 19

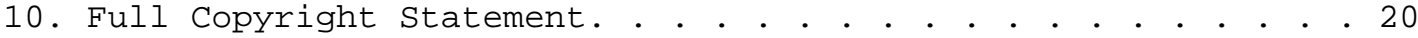




\section{Introduction}

This document defines a MIB module which contains Textual Conventions for Multiprotocol Label Switching (MPLS) networks. These Textual Conventions should be imported by MIB modules which manage MPLS networks.

The key words "MUST", "MUST NOT", "REQUIRED", "SHALL", "SHALL NOT", "SHOULD", "SHOULD NOT", "RECOMMENDED", "MAY", and "OPTIONAL" in this document are to be interpreted as described in RFC 2119 [RFC2119].

For an introduction to the concepts of MPLS, see [RFC3031].

2. The Internet-Standard Management Framework

For a detailed overview of the documents that describe the current Internet-Standard Management Framework, please refer to section 7 of RFC 3410 [RFC3410].

Managed objects are accessed via a virtual information store, termed the Management Information Base or MIB. MIB objects are generally accessed through the Simple Network Management Protocol (SNMP). Objects in the MIB are defined using the mechanisms defined in the Structure of Management Information (SMI). This memo specifies a MIB module that is compliant to the SMIV2, which is described in STD 58, RFC 2578 [RFC2578], STD 58, RFC 2579 [RFC2579] and STD 58, RFC 2580 [RFC2580].

3. MPLS Textual Conventions MIB Definitions

MPLS-TC-STD-MIB DEFINITIONS : := BEGIN

IMPORTS

MODULE-IDENTITY, Unsigned32, Integer32, transmission FROM SNMPV2-SMI $--\quad[R F C 2578]$

TEXTUAL-CONVENTION

FROM SNMPV2-TC;

mplsTCSTdMIB MODULE-IDENTITY

LAST-UPDATED "200406030000Z" -- June 3, 2004

ORGANIZATION

"IETF Multiprotocol Label Switching (MPLS) Working Group." CONTACT-INFO " Thomas D. Nadeau 


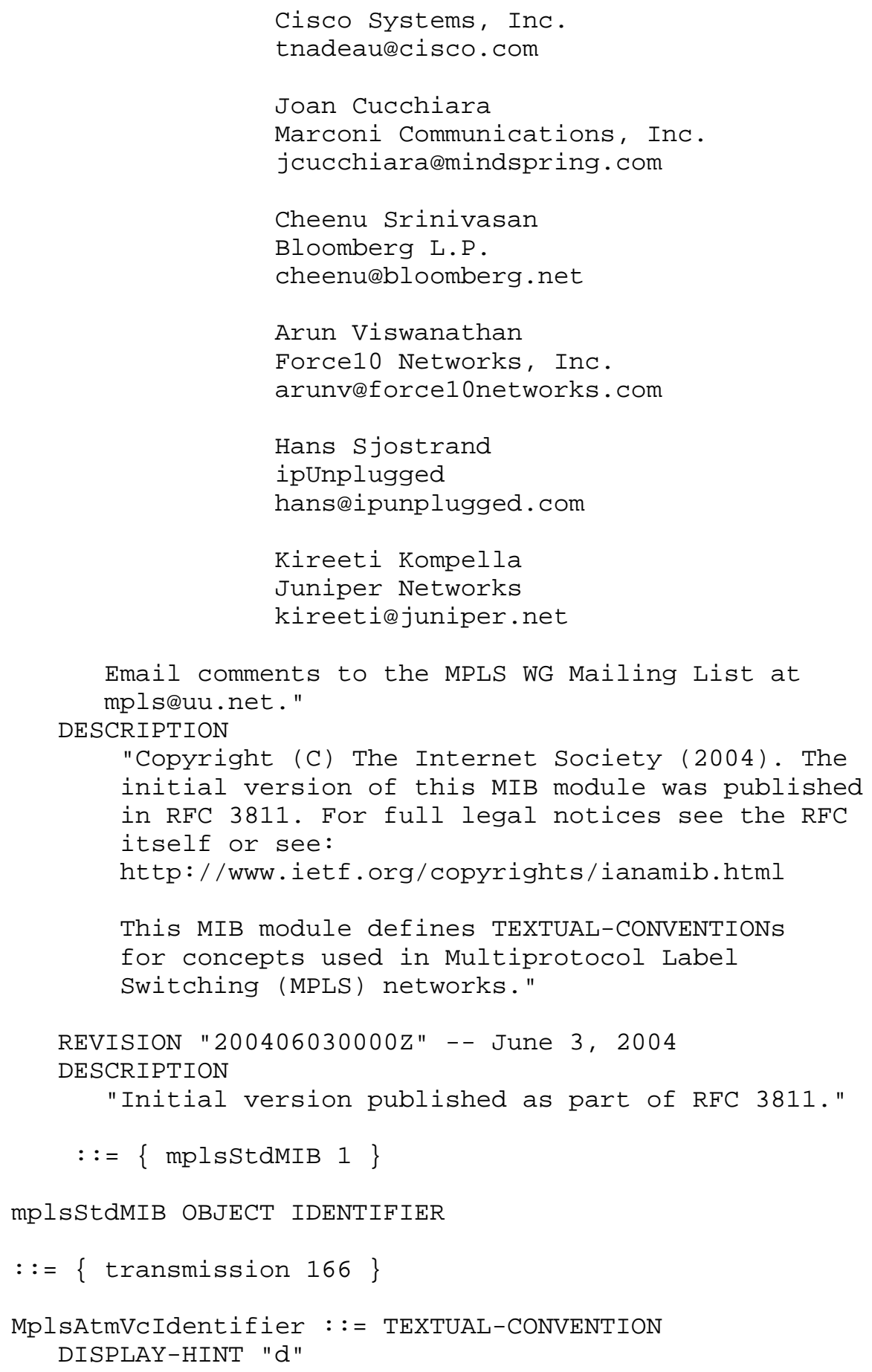




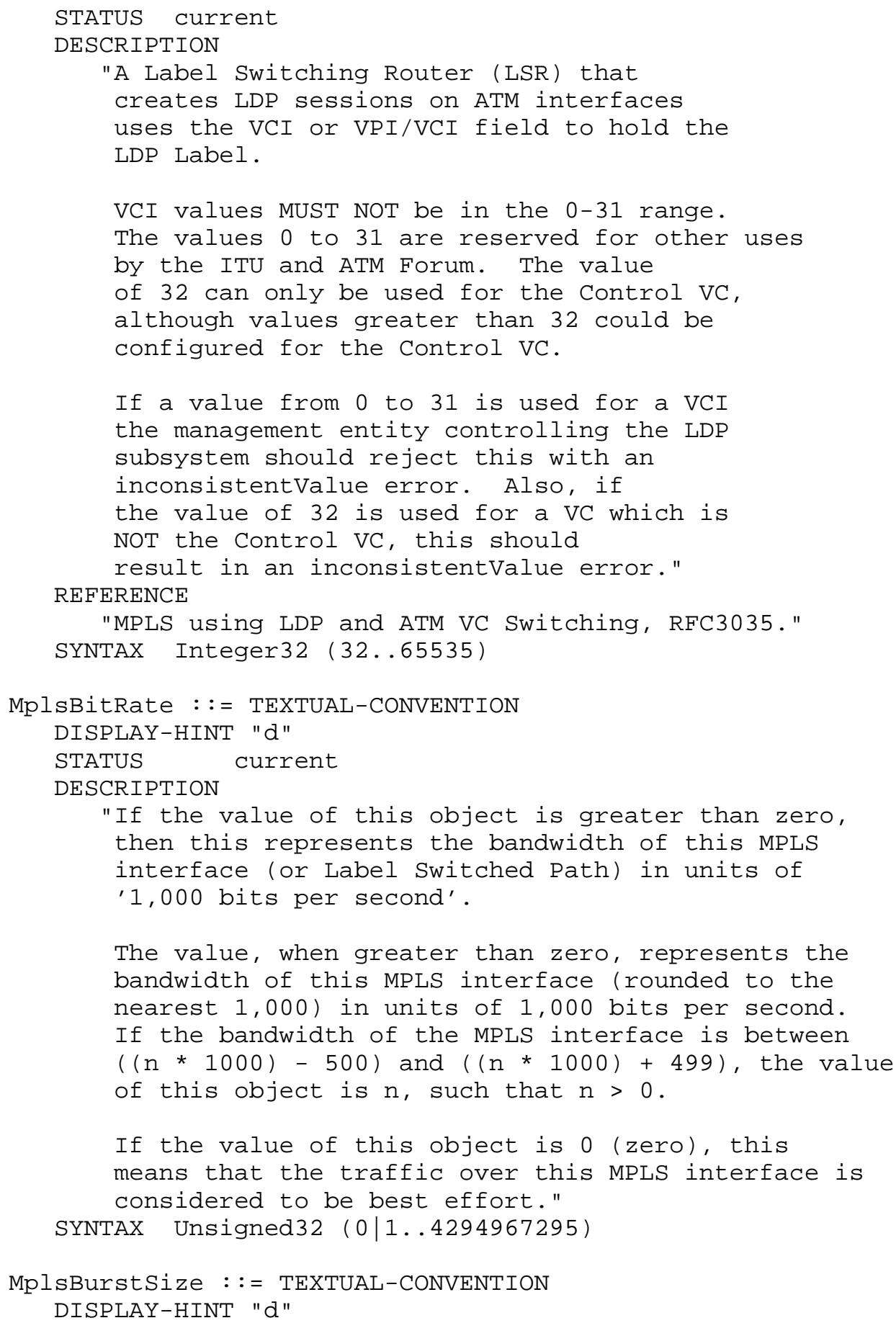




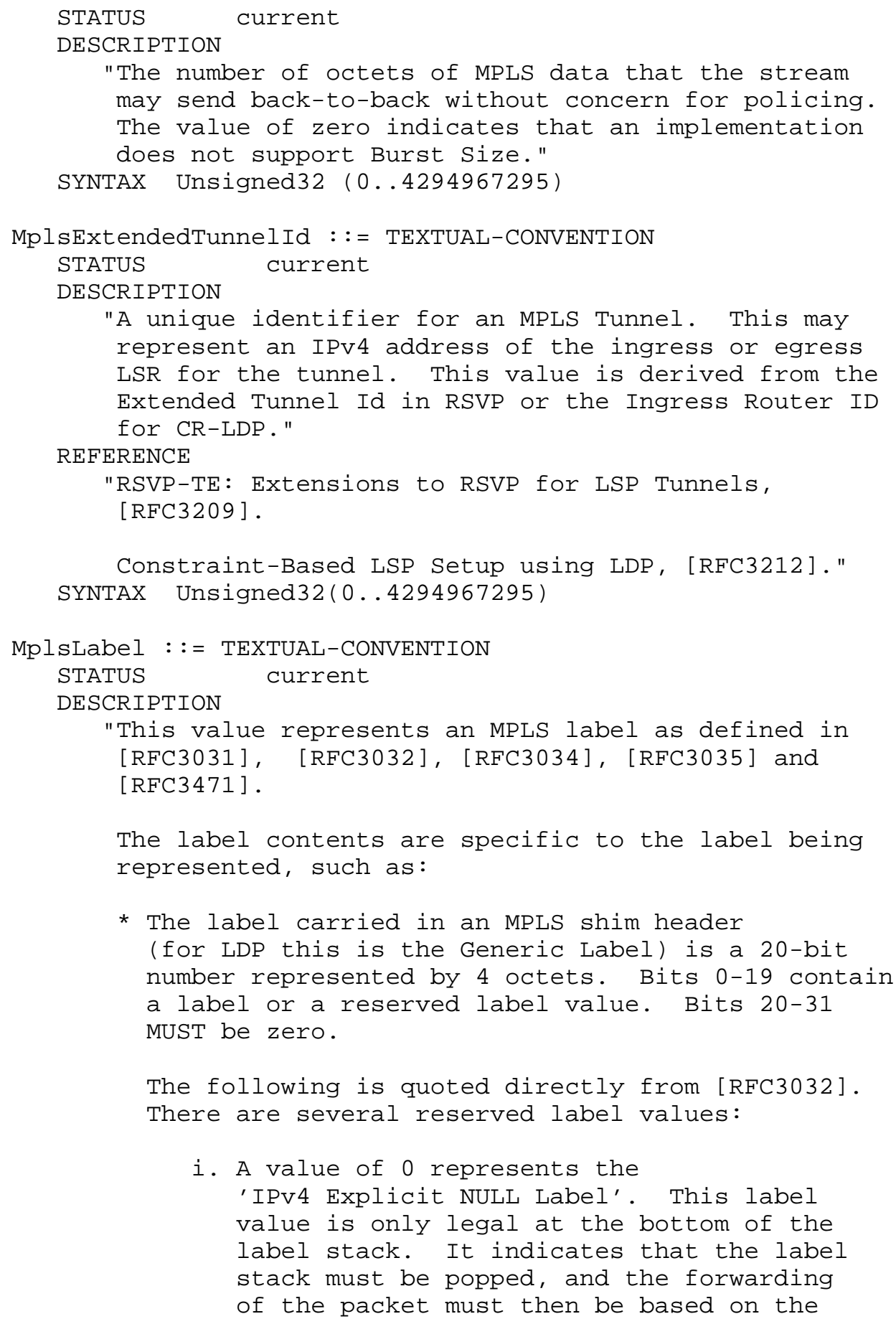


IPv4 header.

ii. A value of 1 represents the

'Router Alert Label'. This label value is

legal anywhere in the label stack except at

the bottom. When a received packet

contains this label value at the top of

the label stack, it is delivered to a

local software module for processing.

The actual forwarding of the packet

is determined by the label beneath it

in the stack. However, if the packet is

forwarded further, the Router Alert Label

should be pushed back onto the label stack

before forwarding. The use of this label

is analogous to the use of the

'Router Alert Option' in IP packets

[RFC2113]. Since this label

cannot occur at the bottom of the stack,

it is not associated with a

particular network layer protocol.

iii. A value of 2 represents the

'IPv6 Explicit NULL Label'. This label

value is only legal at the bottom of the

label stack. It indicates that the label

stack must be popped, and the forwarding

of the packet must then be based on the

IPv6 header.

iv. A value of 3 represents the

'Implicit NULL Label'.

This is a label that an LSR may assign and

distribute, but which never actually

appears in the encapsulation. When an

LSR would otherwise replace the label

at the top of the stack with a new label,

but the new label is 'Implicit NULL',

the LSR will pop the stack instead of

doing the replacement. Although

this value may never appear in the

encapsulation, it needs to be specified in

the Label Distribution Protocol, so a value is reserved.

v. Values 4-15 are reserved.

* The frame relay label can be either 10-bits or 


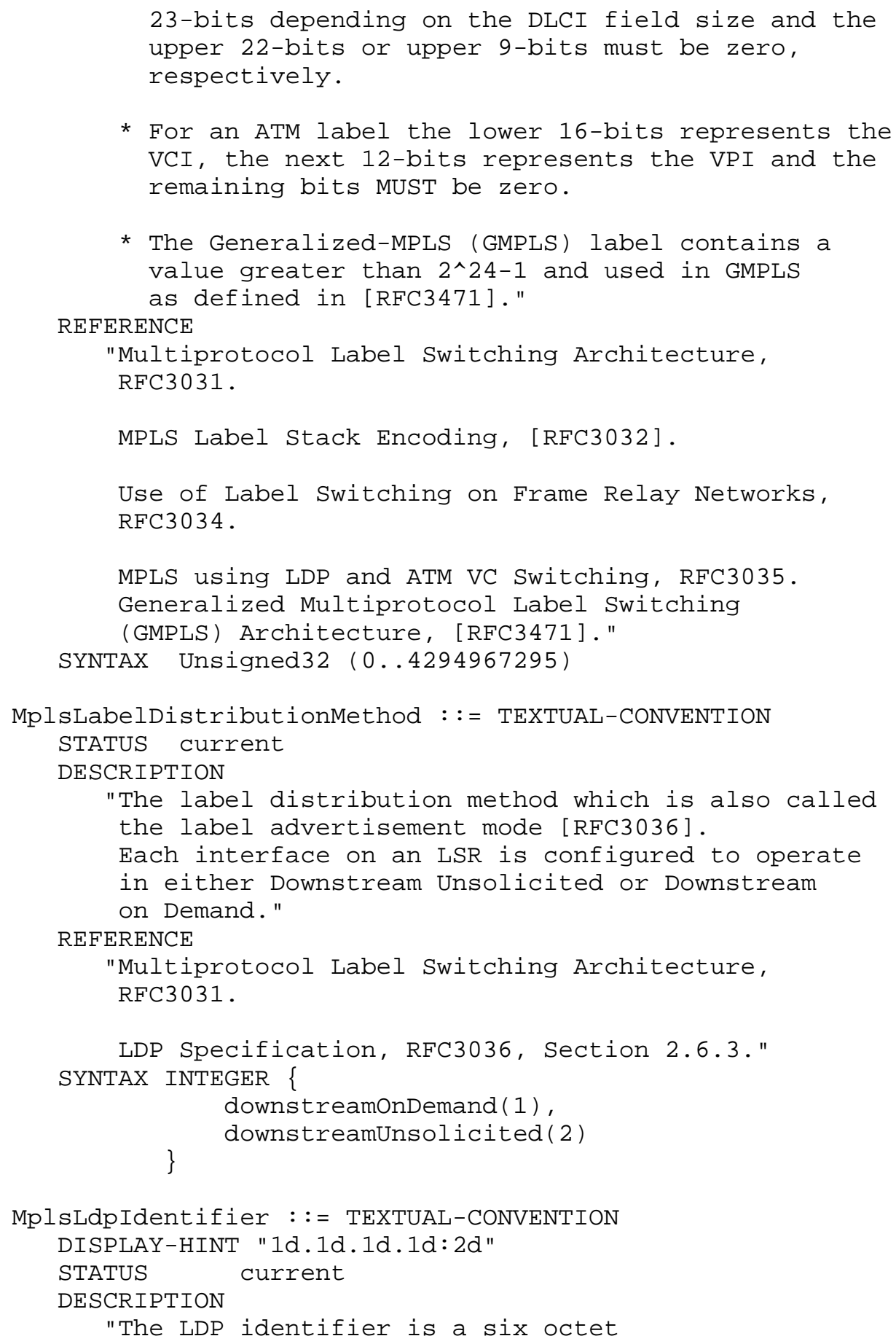

"Multiprotocol Label Switching Architecture, RFC3031.

MPLS Label Stack Encoding, [RFC3032].

Use of Label Switching on Frame Relay Networks, RFC3034.

MPLS using LDP and ATM VC Switching, RFC3035. Generalized Multiprotocol Label Switching (GMPLS) Architecture, [RFC3471]." 


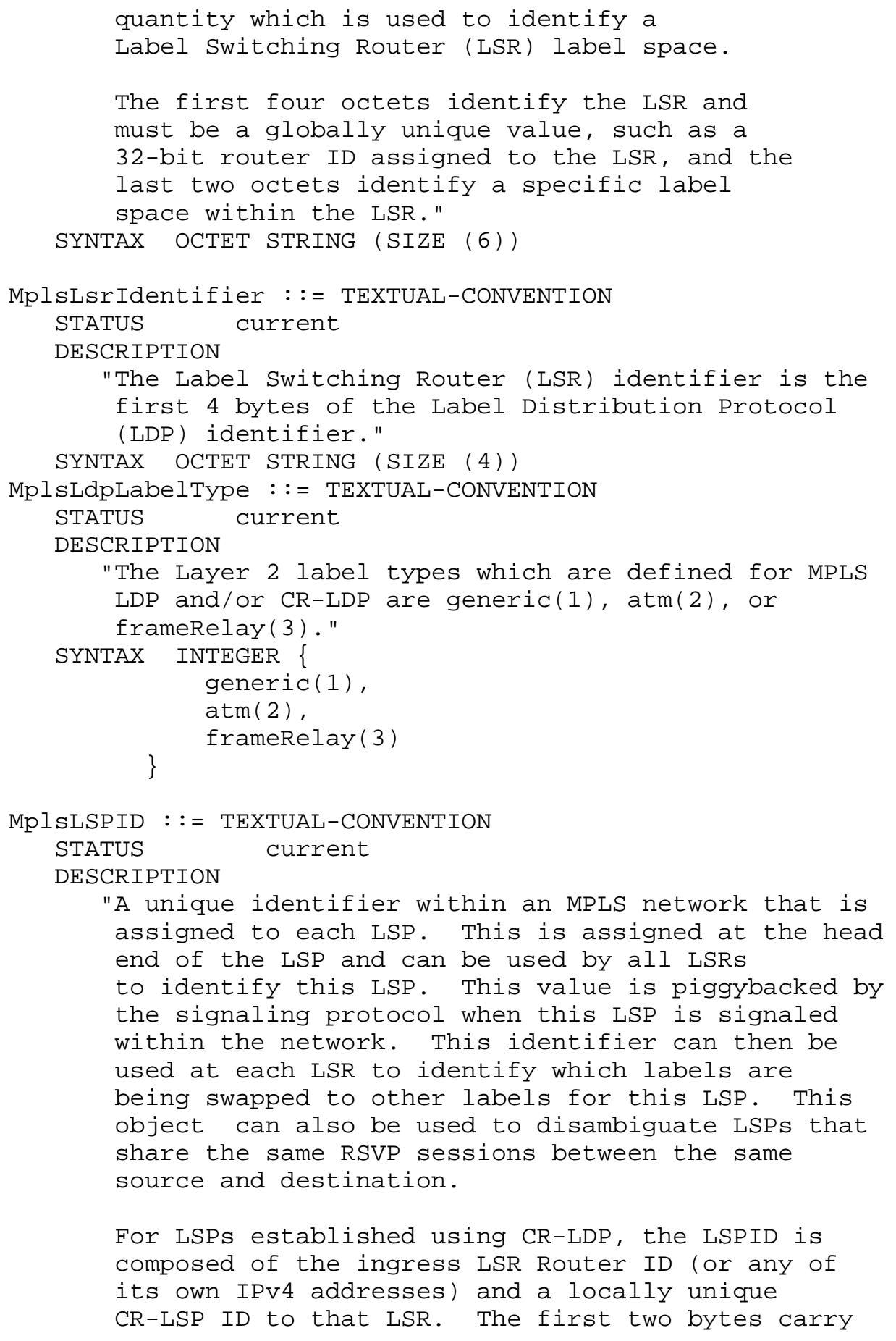




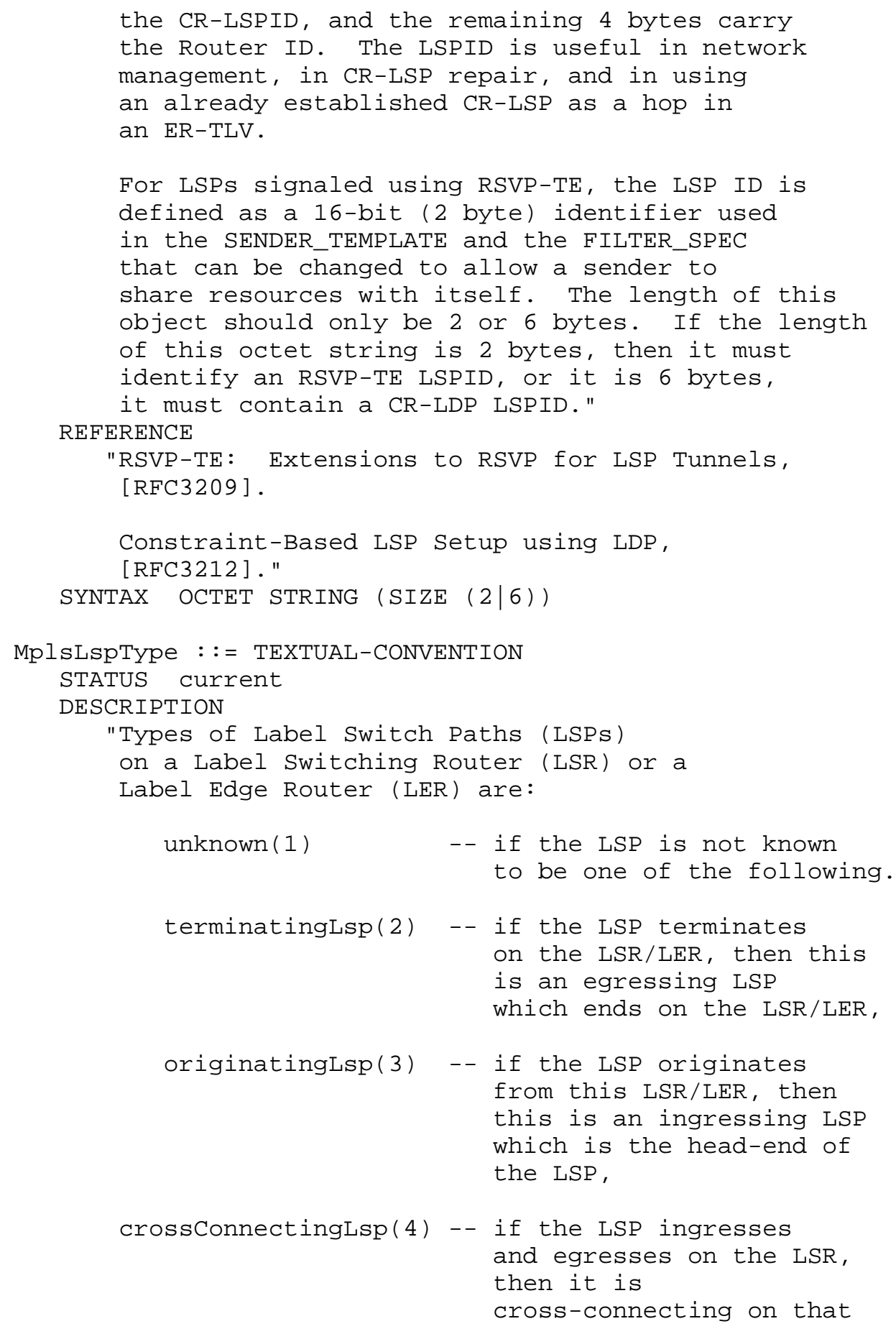




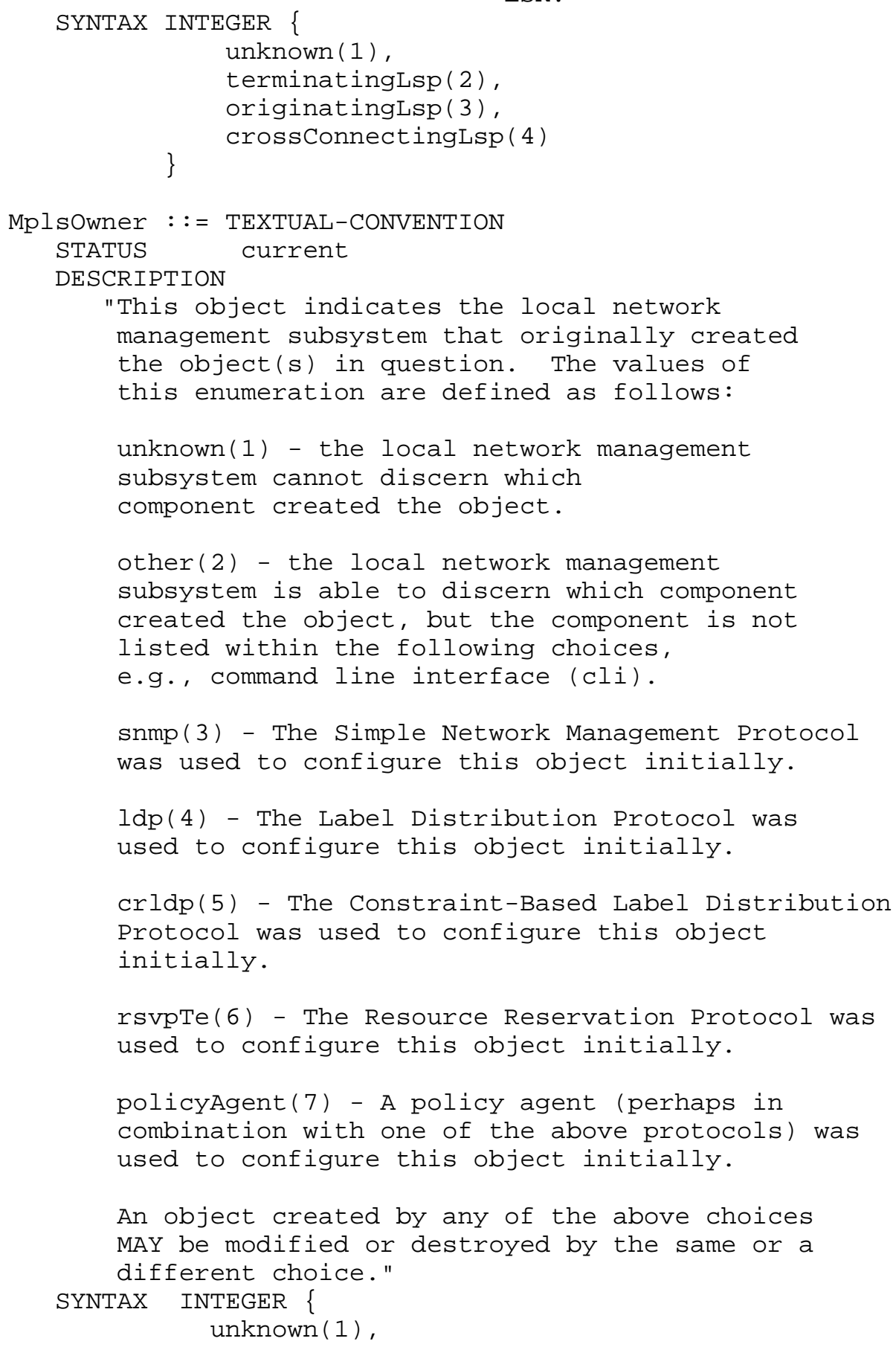




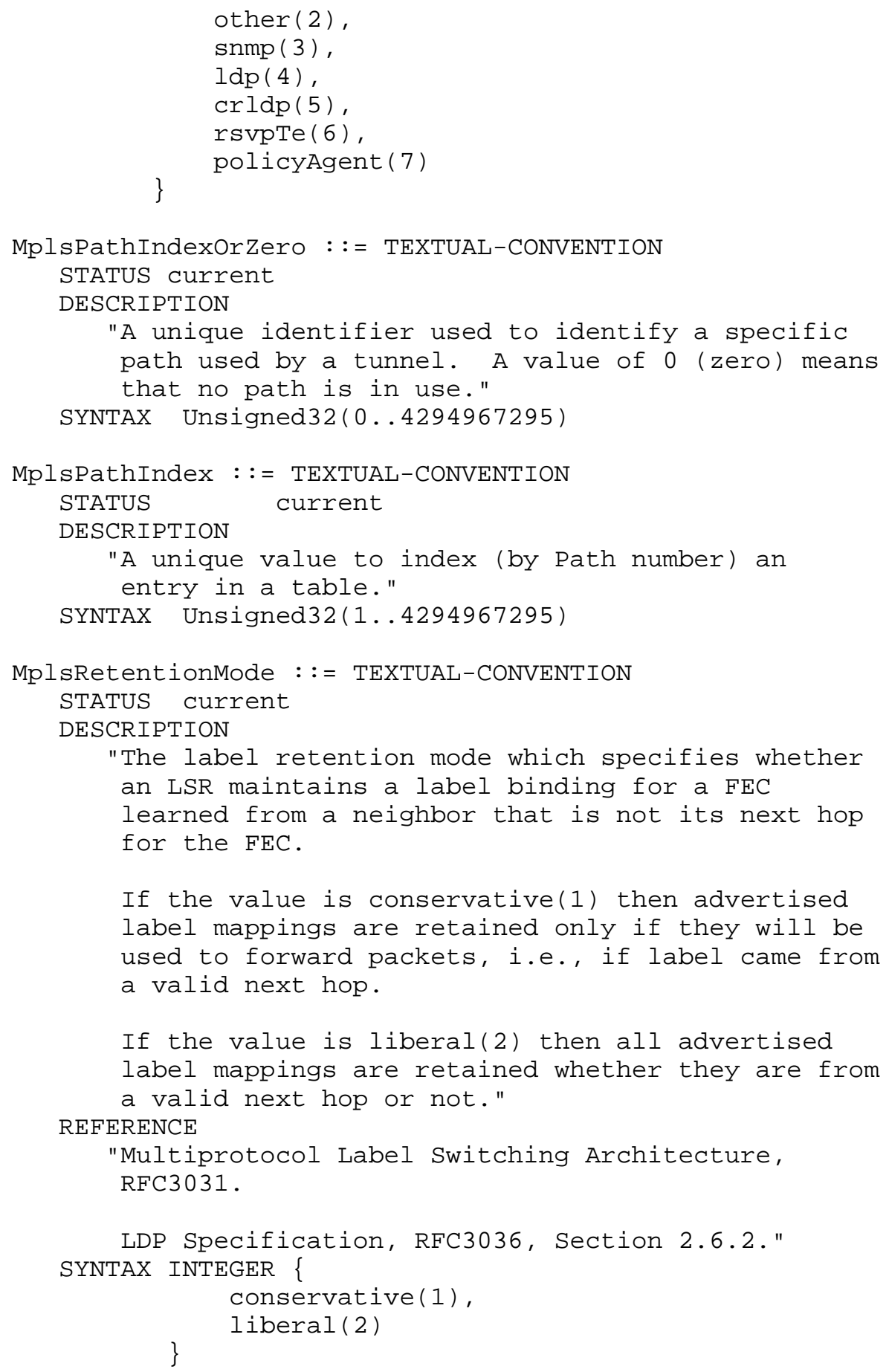




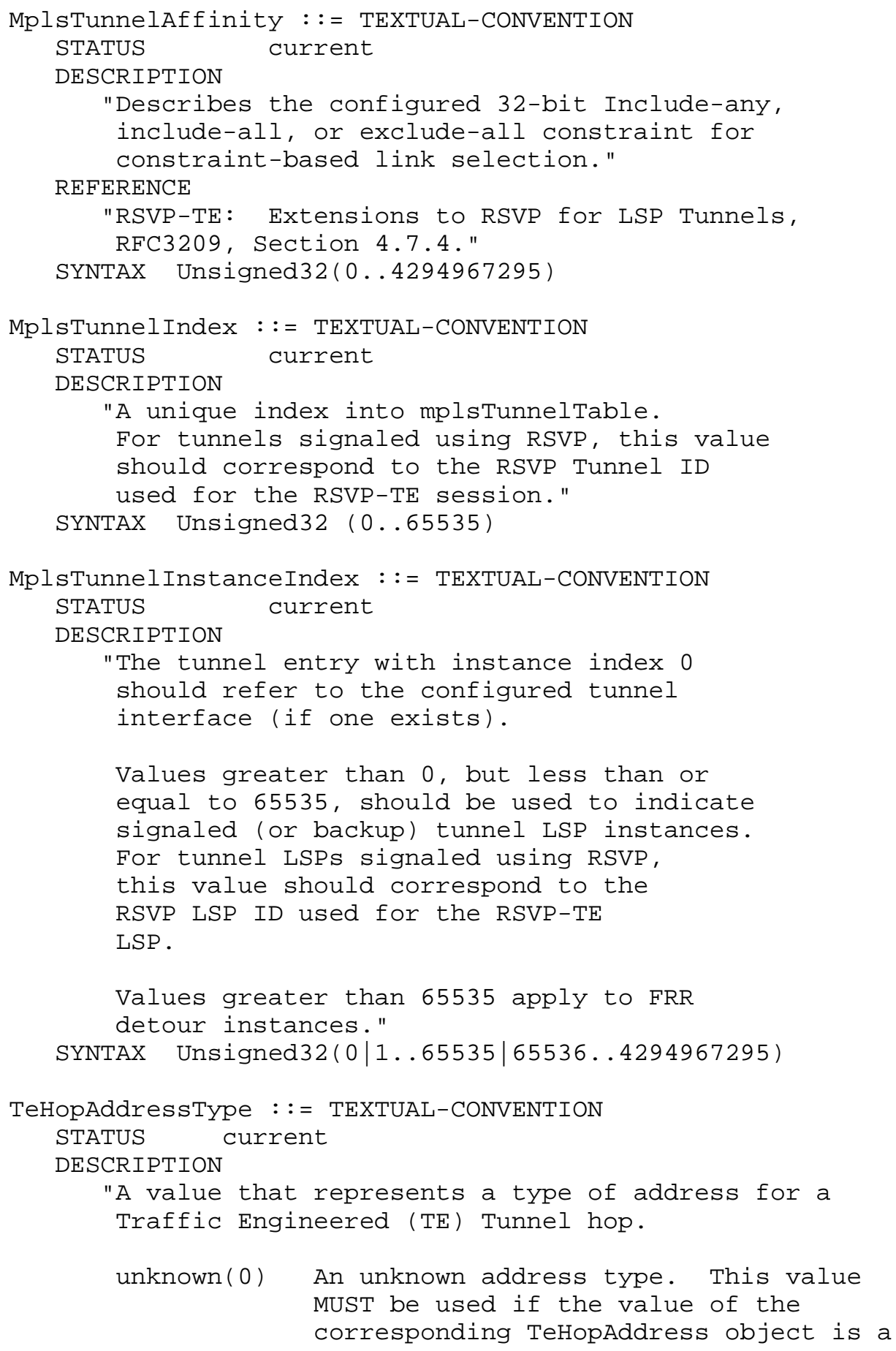




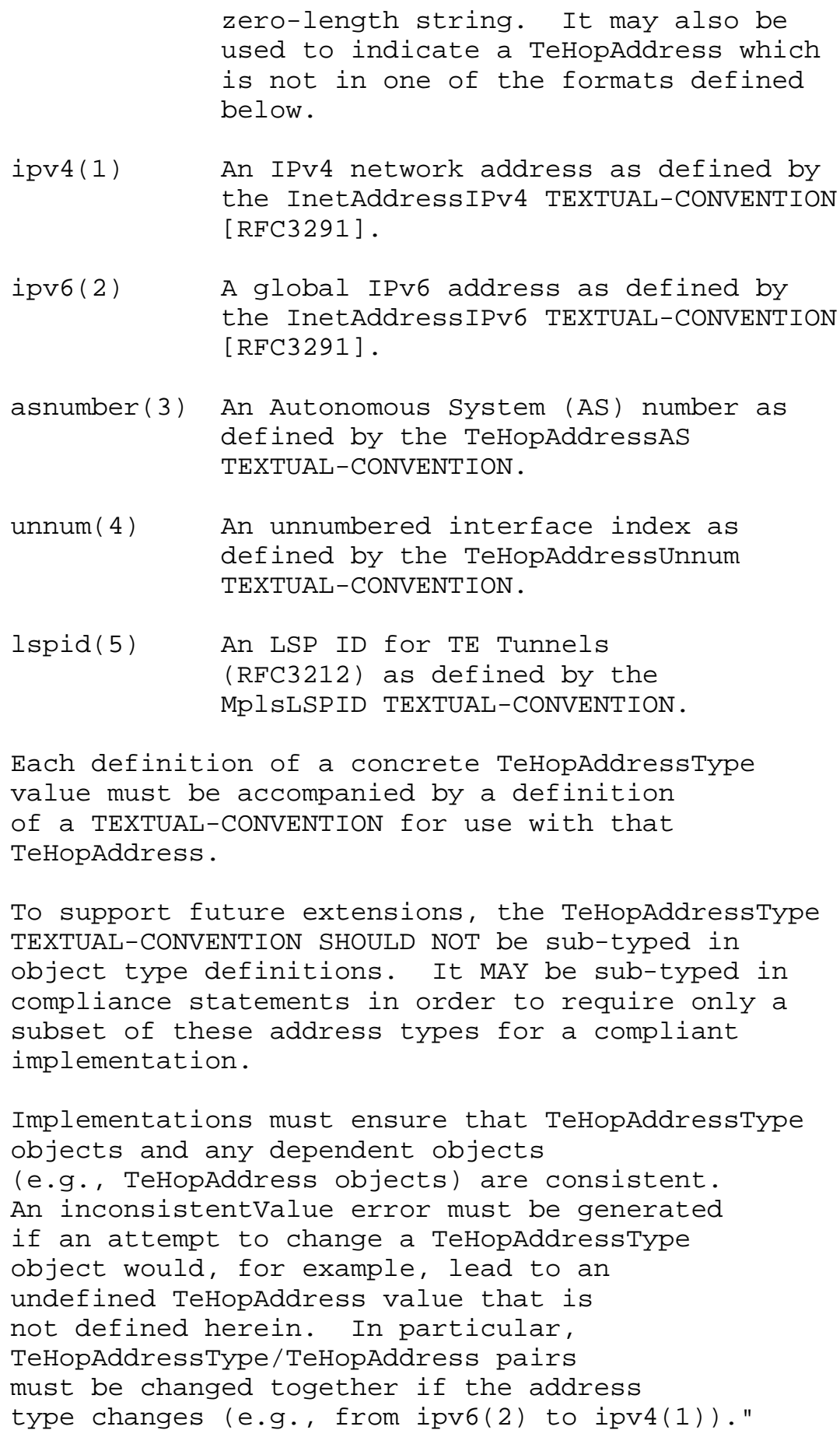




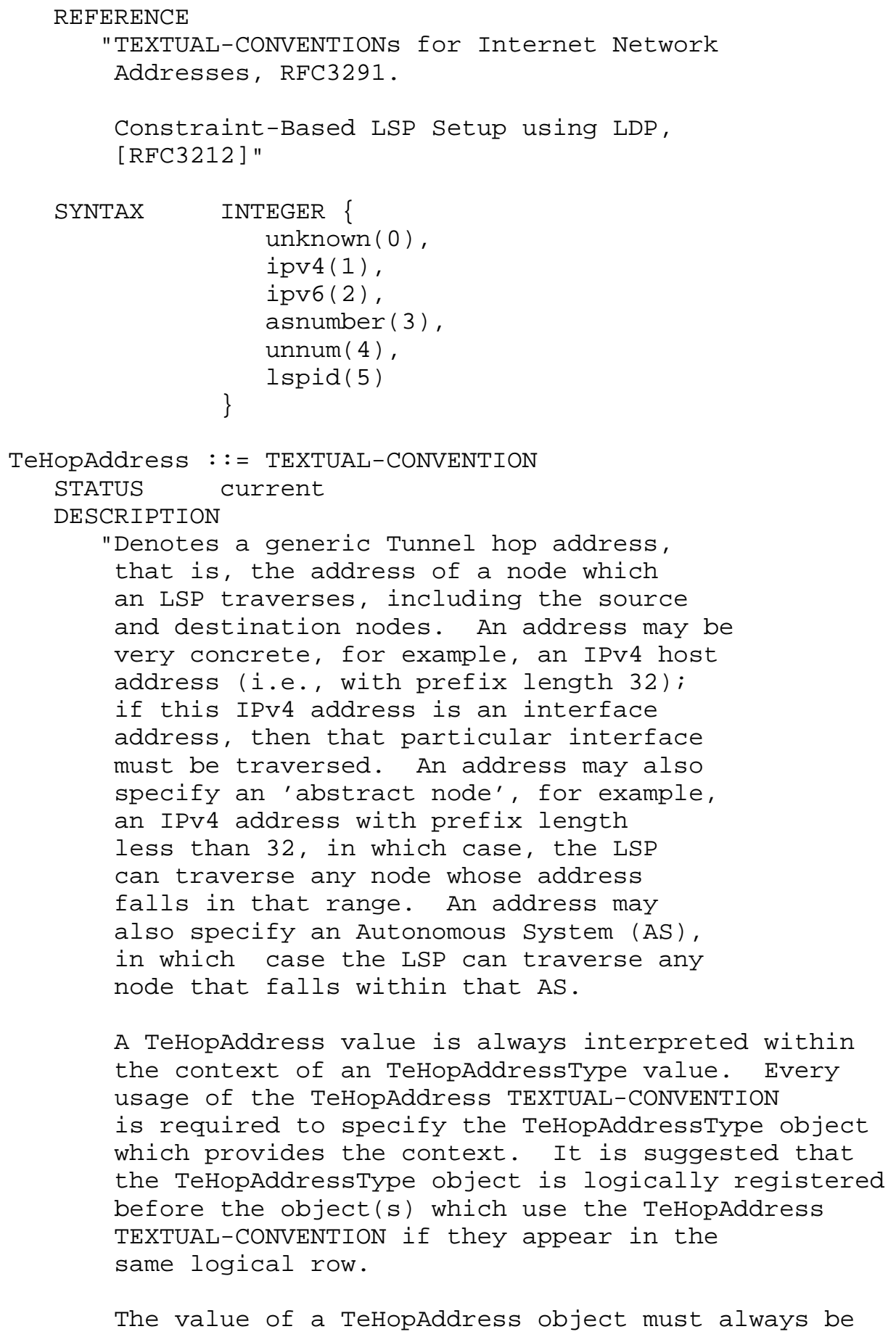




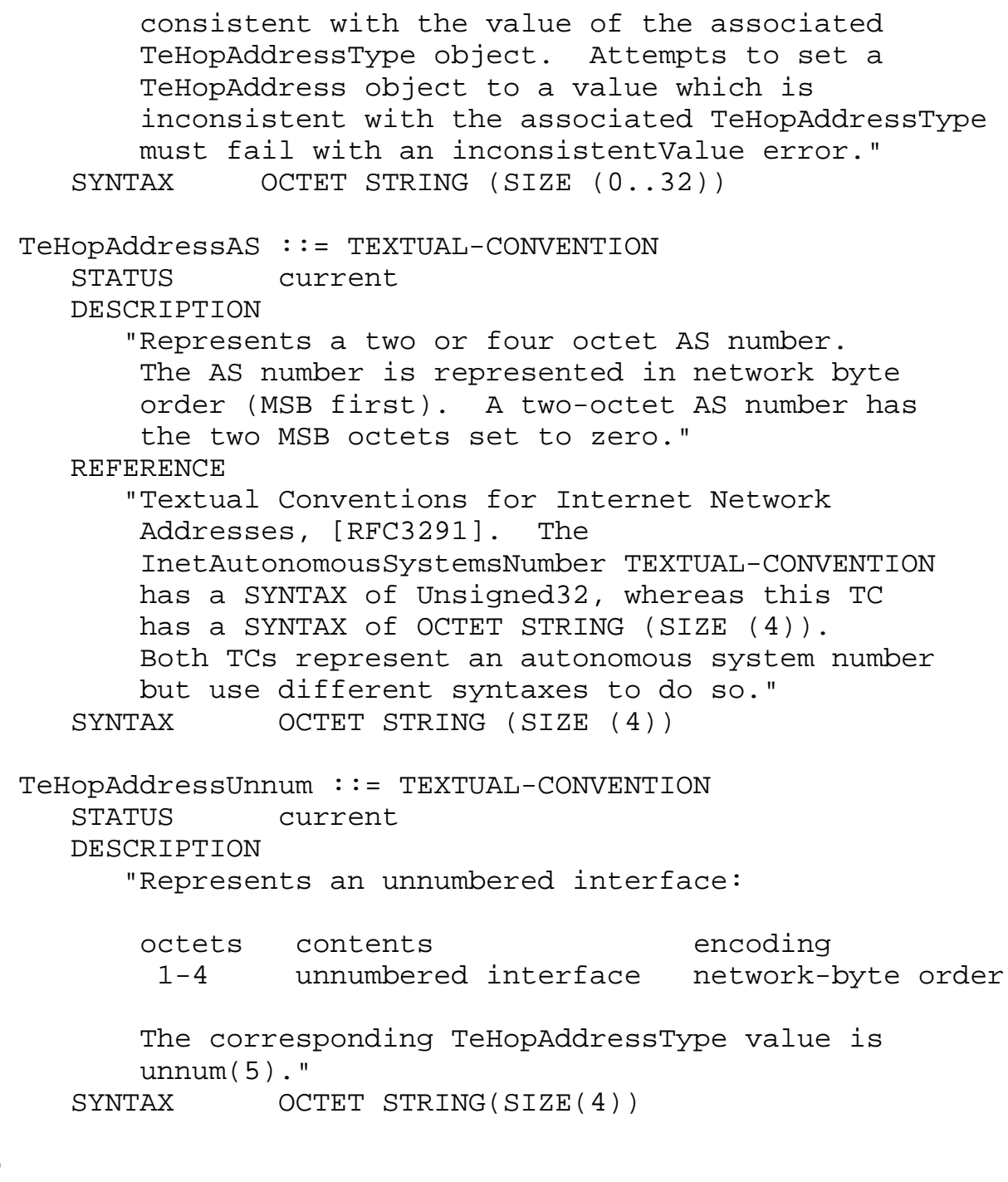

END 


\section{References}

\subsection{Normative References}

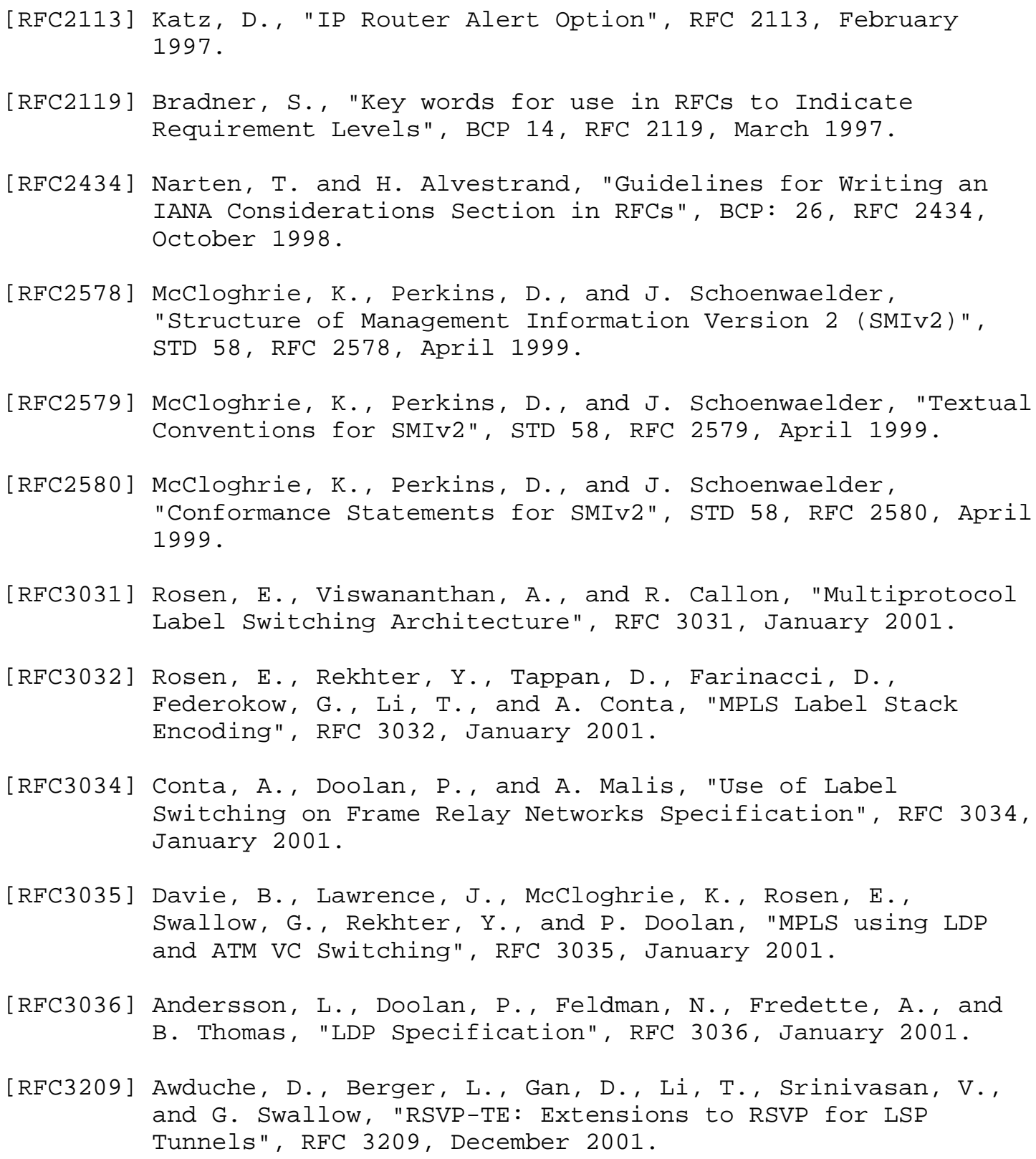


[RFC3212] Jamoussi, B., Ed., Andersson, L., Callon, R., Dantu, R., Wu, L., Doolan, P., Worster, T., Feldman, N., Fredette, A., Girish, M., Gray, E., Heinanen, J., Kilty, T., and A. Malis, "Constraint-Based LSP Setup using LDP", RFC 3212, January 2002 .

[RFC3291] Daniele, M., Haberman, B., Routhier, S., and J. Schoenwaelder, "Textual Conventions for Internet Network Addresses", RFC 3291, May 2002.

[RFC3471] Berger, L., Editor, "Generalized Multi-Protocol Label Switching (GMPLS) Architecture", RFC 3471, January 2003.

\subsection{Informative References}

[RFC3410] Case, J., Mundy, R., Partain, D., and B. Stewart, "Introduction and Applicability Statements for InternetStandard Management Framework", RFC 3410, December 2002.

5. Security Considerations

This module does not define any management objects. Instead, it defines a set of textual conventions which may be used by other MPLS MIB modules to define management objects.

Meaningful security considerations can only be written in the MIB modules that define management objects. Therefore, this document has no impact on the security of the Internet.

\section{IANA Considerations}

IANA has made a MIB OID assignment under the transmission branch, that is, assigned the mplsstdMIB under \{transmission 166 . This sub-id is requested because 166 is the iftype for mpls(166) and is available under transmission.

In the future, MPLS related standards track MIB modules should be rooted under the mplsStdMIB subtree. The IANA is requested to manage that namespace. New assignments can only be made via a Standards Action as specified in [RFC2434].

The IANA has also assigned $\{$ mplsStdMIB 1$\}$ to the MPLS-TC-STD-MIB specified in this document. 


\section{Contributors}

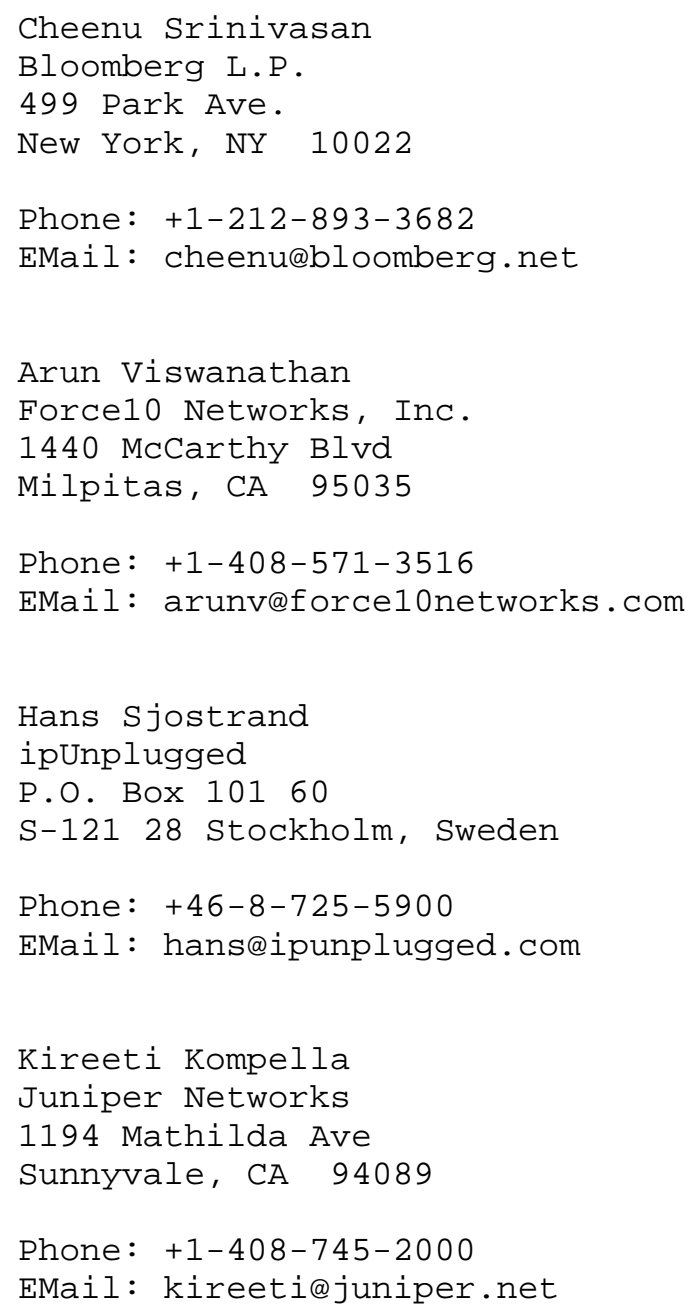




\section{Acknowledgements}

This document is a product of the MPLS Working Group. The editors and contributors would like to thank Mike MacFadden and Adrian Farrel for their helpful comments on several reviews. Also, the editors and contributors would like to give a special acknowledgement to Bert Wijnen for his many detailed reviews. Bert's assistance and guidance is greatly appreciated.

9. Authors' Addresses

Thomas D. Nadeau

Cisco Systems, Inc.

BXB300/2/

300 Beaver Brook Road

Boxborough, MA 01719

Phone: +1-978-936-1470

EMail: tnadeaulcisco.com

Joan E. Cucchiara

Marconi Communications, Inc.

900 Chelmsford Street

Lowell, MA 01851

Phone: +1-978-275-7400

EMail: jcucchiara@mindspring.com 
10. Full Copyright statement

Copyright (C) The Internet society (2004). This document is subject to the rights, licenses and restrictions contained in BCP 78, and except as set forth therein, the authors retain all their rights.

This document and the information contained herein are provided on an "AS IS" basis and THE CONTRIBUTOR, THE ORGANIZATION HE/SHE REPRESENTS OR IS SPONSORED BY (IF ANY), THE INTERNET SOCIETY AND THE INTERNET ENGINEERING TASK FORCE DISCLAIM ALL WARRANTIES, EXPRESS OR IMPLIED, INCLUDING BUT NOT LIMITED TO ANY WARRANTY THAT THE USE OF THE INFORMATION HEREIN WILL NOT INERINGE ANY RIGHTS OR ANY IMPLIED WARRANTIES OF MERCHANTABILITY OR FITNESS FOR A PARTICULAR PURPOSE.

Intellectual Property

The IETF takes no position regarding the validity or scope of any Intellectual Property Rights or other rights that might be claimed to pertain to the implementation or use of the technology described in this document or the extent to which any license under such rights might or might not be available; nor does it represent that it has made any independent effort to identify any such rights. Information on the procedures with respect to rights in RFC documents can be found in BCP 78 and BCP 79 .

Copies of IPR disclosures made to the IETF Secretariat and any assurances of licenses to be made available, or the result of an attempt made to obtain a general license or permission for the use of such proprietary rights by implementers or users of this specification can be obtained from the IETF on-line IPR repository at http://www.ietf.org/ipr.

The IETF invites any interested party to bring to its attention any copyrights, patents or patent applications, or other proprietary rights that may cover technology that may be required to implement this standard. Please address the information to the IETF at ietfipreietf.org.

Acknowledgement

Funding for the RFC Editor function is currently provided by the Internet Society. 